\title{
MASTICAR: Cómo la evidencia muestra que la Fuerza Motriz Contribuye en el Desarrollo Facial
}

\author{
Licia Paskay ${ }^{3}$ \\ Presidente de la sin-fines-de-lucro Academia de Ciencias Aplicadas miofuncionales (AAMS). \\ Miembro de la Junta de instructores de la Academia de Terapia Miofuncional Orofacial (AOMT). \\ En la práctica privada y contratista independiente desde 2002, trabajando con personas con trastornos \\ neurológicos y con trastornos miofuncionales.
}

\section{ABSTRACT: English}

Chewing is still a neglected aspect of therapy and health, although the muscles and soft tissue connected to them are known to guide the growth, development and maintenance of the orofacial boney structures, through a process called epigenetic. Epigenetics is the process in which the environment provides, or not, the necessary stimuli for optimal craniofacial functions and development by impacting the expression of the individual DNA. In the course of human history, changes in food consistency, through cooking, industrial processing and cultural habits, changed chewing, contributing to a phenomenon called klinorhynchy, or a downward and backward growth vector of the orofacial structures, now related to airway issues and dental malocclusion. Chronic reduction or absence of chewing, since weaning, may impact many aspects of health in children and adults.

\section{Spanish}

Los expertos en la odontología evolutiva han documentado durante un tiempo que la raza humana ha pasado por cambios muy rápidos en la estructura facial ya que la "invención" de la cocina y de la agricultura comenzó hace unos 12.000 años. Sumando a esto una aceleración significativa en la evolución de la cara humana ha tenido lugar, posiblemente debido a la preparación y consumo de alimentos modernos (como pasta, comida rápida, alimentos procesados, etc). La aceleración de este movimiento evolutivo (llamado klinorhynchy) de la cara y la lengua hacia la parte posterior del cráneo, tiene implicaciones para obstruir las vías respiratorias, agravando y creando los trastornos del sueño y otros desordenes craneofaciales.

Mediante la optimización de las funciones musculares orofaciales, y el impacto de los tejidos blandos directamente en el crecimiento y desarrollo de los huesos de la cara, boca, cuello, hombros, etc., tienen una interacción recíproca de forma y función. Lo que comemos, cómo comemos y cuándo lo comemos tiene un impacto directo en las estructuras duras (huesos y dientes) a través de un proceso llamado epigenética.

Estudios recientes en modelos animales, así como en seres humanos han identificado múltiples beneficios de mascar, como mejor digestión y oclusión dental, reducción del estrés, un aumento del flujo sanguíneo cerebral, aumento de la atención y de la concentración.

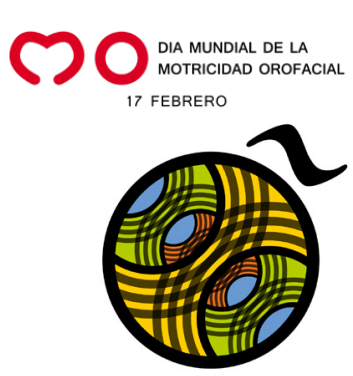

3. Presidente de la sin-fines-de-lucro Academia de Ciencias Aplicadas miofuncionales (AAMS). Miembro de la Junta de e instructor de la Academia de Terapia Miofuncional Orofacial (AOMT). En la práctica privada y contratista independiente desde 2002, trabajando con personas con trastornos neurológicos y con trastornos miofuncionales. 
Teniendo en cuenta todos los beneficios, la masticación debe encontrar una posición más importante y practica en la terapia orofacial, comenzando con los cambios en las prácticas de destete, motivar niños, adultos y ancianos a mejorar la masticación, porque la masticación es una manera sencilla y eficaz de prevenir algunos dismorfismos faciales y de promover mejores funciones orofaciales como la deglución, la oclusión dental y la eficiencia de comer.

\section{TYPE OF ARTICLE: Clinical Literature Review}

KEYWORDS: Chewing, epigenetics, matrix, klinorhynky, weaning

\section{Contact info:}

Licia Coceani Paskay

5800-A Hannum Avenue, Suite 225

Culver City, California 90230 USA

Icpaskay@gmail.com

+1-310-351-4084

\section{Licia Coceani Paskay, MS, CCC-SLP}

La masticación se ha descuidado en la terapia; y los estudios en los últimos 10 a 20 años están apoyando este concepto. Para entender mejor el impacto de la masticación en el rostro humano y la boca, tenemos que considerar lo que la falta de mascar nos ha estado causando. Nuestros antepasados tenían bocas grandes, posicionadas delante de la nariz, con mucho espacio para la lengua y un amplio espacio para las vías respiratorias.

Luego, con el tiempo, debido los alimentos cocinados más suaves, nuestras caras han estado emigrando hacia abajo y atrás (Varrela, 1992; Lieberman, 2011), un fenómeno llamado klinorhynky (Schumacher et al, 1994). Klinorhynchy restringe el volumen oral causando que la lengua invada las vías respiratorias, especialmente en la noche, causando apnea del sueño.

El proceso responsable de klynorhynchy se llama epigenética que es una rama nueva de la biología y que va en expansión (Francis, 2011; Hallgrimmson \& Hall, 2011). La epigenética es el principio que permite controles en la expresión de nuestros genes, debido a las demandas en el medio ambiente, sin cambiar el propio ADN. Por ejemplo, al igual que puedo decir hola (que es la información genética) en dos o más formas (hola o hola), por lo que, nuestros genes producen varias proteínas o moléculas dependiendo de lo que necesite la demanda del medio ambiente (epigenética).

Una fuente de información general sobre el concepto de la epigenética y sobre algunos aspectos de mascar vienen de los libros de Daniel Lieberman y de Roberto Corruccini $(1984,1990)$ en particular, que son investigadores importantes en los EE.UU. Ellos explican la "evolución" o "deevolución" de la cara humana debido a la falta de mascar y a los cambios en la producción de los alimentos y la alimentación. Melvin Moss también, en el '97, escribió cuatro artículos que explican por qué la masticación es tan importante en el desarrollo del complejo craneofacial (y nasal). La mayoría de los conceptos que son presentados a continuación sobre epigenética son tomados de estos artículos.

La epigenética implica todos los cambios debido al ambiente externo al organismo (o células) y todos los cambios dentro del organismo o células y dentro del espacio extracelular. Todas las plantas responden a los efectos epigenéticos de su entorno porque no pueden moverse así que 
tienen que adaptarse constantemente a la sequía, la lluvia, el frío etc. Los animales también dependen de influencias epigenéticas, que les permitan adaptarse a los cambios en el medio ambiente y la dieta. El estudio más conocido en el impacto de la epigenética del medio ambiente en los seres humanos se llama el estudio de cohortes hambruna holandesa, en el que se estudió el efecto de la inanición en la 2da guerra mundial por ahora a 4 generaciones, y los efectos de esta experiencia aún están presentes en la expresión genética de los descendientes de aquellas mujeres embarazadas que tenían hambre (Francis, 2011).

Para resumir el concepto de la matriz funcional, o cómo se modifican los huesos, Melvin Moss declaró que son los tejidos blandos (músculos, tendones, periorstium, ligamentos, etc.) que modifican la forma, posición, crecimiento, desarrollo, mantenimiento y cualquier otro cambio en los huesos, sin excepciones. Ningún hueso está expuesto al ambiente externo. Todos están cubiertos por los tejidos blandos.

Todas las células, incluyendo las células óseas son irritables y responden al estímulo con dos grandes tipos de respuestas: una deformación mecánica y una transducción eléctrica, al igual que cuando se utiliza el interruptor de la luz, mecánicamente, el impulso eléctrico causa que la bombilla se apague o encienda.

Las células óseas y el tejido óseo responden a una carga (empujar o tirar de los tejidos blandos / o aparatos de ortodoncia) con tres respuestas diferentes: Las células óseas aumentan (deposición), disminuyen (reabsorción) o permanecen igual (mantenimiento). Este tipo de respuesta es única de el hueso.

El tejido óseo tiene otras características: las células sensoriales del hueso no son especializadas como otras células sensoriales (por ejemplo, las células sensoriales de la yema del dedo). Las células sensoriales de la yema del dedo sólo tienen una respuesta, dependiendo de la célula (como dolor, calor, el empuje; el estiramiento) mientras que el tejido óseo puede tener tres respuestas (reabsorción, deposición y mantenimiento). Todo esto sin la participación de los nervios ya que el tejido óseo es aneural y debe comunicarse con el resto del cuerpo por medio de los tejidos blandos que lo envuelve.

Al igual que las neuronas en el cerebro, las células óseas están conectados en forma de brechas entre ellos, similar a la brecha sináptica, excepto que el impulso es bidireccional, desde la célula a otras células y viceversa. Todas las células óseas, excepto los osteoclastos tienen esa capacidad, y porque cada célula es eléctricamente activa, cada hueso es un campo eléctrico. Por otra parte, las células se auto-organizan en unidades de trabajo, similar a una colmena de abejas o de un hormiguero o un banco de peces, donde los pequeños individuos tienen sus propias vidas, pero el trabajo en conjunto hacen una función más grande.

Debido a esta independencia y auto-organización, las células óseas "saben" qué tipo de señal necesitan para generar una respuesta para una carga: algunas respuestas son activas y algunas son inhibitorias, al igual que lo hace nuestro cerebro. A medida que la carga muscular cambia por alguna razón, el hueso cambiará en consecuencia.

La carga que se aplica por los tejidos blandos en los tejidos óseos, que son intermitentes, con el tiempo y con una cierta intensidad, deformará el hueso de una manera u otra. Esto debido a que el tejido blando es el que "habla" con el tejido duro. Este es un mecanismo similar al que las ondas sonoras se transforman en impulsos eléctro/neuroquímicos por el oído interno.

Los estudios realizados en la relación entre los músculos y los huesos demuestran que el músculo 
tiene una cierta frecuencia, pequeña / aproximadamente $2 \mathrm{~Hz}$, pero es suficiente para generar una respuesta biológica. Lo sorprendente es que el hueso debajo del tejido blando (músculo corresponsal) responderá a la frecuencia exacta, como si los huesos y músculos hablaran el mismo idioma.

Las cargas mecánicas cambian la expresión del ADN. Sabemos que estamos diseñados físicamente para soportar la gravedad de la Tierra, pero también estamos diseñados para ser capaces de utilizar nuestros músculos para trabajar y hacer deportes. Cambiamos la masa muscular y su uso porque hay una cadena molecular que cruza cada célula ósea desde la membrana hacia varios sitios en los genes y provoca cambios en las moléculas duplicadas por el ARN mensajero. Esta red de vías moleculares hacen la comunicación ADN/medio ambiente, recíproca, contínua y dinámica.

Este mecanismo que permite una respuesta del ADN a las demandas de afuera se llama metilación y es el tema de muchos estudios de investigación, especialmente en oncología. Así que hay un flujo constante de información molecular desde y hacia el medio ambiente y el núcleo de cualquier célula ósea (la médula), aunque esto representado es sólo una célula estándar. Pero los principios se aplican a la célula ósea también.

Con un cerebro sano, que envía las señales adecuadas a los músculos, podemos afectar el fenotipo o la manera en que nos vemos. Lo mismo sucede cuando el cerebro no funciona, los músculos tienen la información incorrecta o insuficiente y el hueso se deforma también como resultado de los tejidos blandos defectuosos.

Tanto para los tejidos blandos y duros, los factores epigenéticos afectan el crecimiento, desarrollo y mantenimiento de los huesos y los músculos correspondientes.Lo que es sorprendente es que el tejido óseo se auto-regula y lo hace mientras permanece en constante comunicación con los tejidos blandos que están dirigidos por los nervios y coordinados por el cerebro.

Asi que, yendo a algo específico, ¿cómo se manifesta esta relación tejido blando/huesos? La manera más importante es masticando. Masticar provee el contacto intermitente, dinámico y recíproco entre los tejidos blandos (especialmente los músculos) y los huesos.

Entonces, ¿qué hay de nuevo en el campo de mascar? En la mayoria de los países, donde están comiendo los alimentos locales no procesados pueden estar bien; pero en los EE.UU. y otros países del Oeste, tenemos epidemias causadas por demasiados alimentos procesados y comida rápida.

Sin embargo, aquí hay un estudio (Katsaros et al., 2006) realizado en ratones (como la gran mayoría de los estudios de este tipo), donde los ratones fueron destetados ya sea con alimentos duros o alimento líquido suave. El grupo de ratones adultos que comió el alimento duro tenían un hueso palatal más grueso al nivel de la sutura.

El estudio de Mavropoulos et al. 2010, demostró que si los ratones fueron alimentados con comida blanda y más tarde fueron introducidos a alimentos duros, la estructura del hueso mandibular mejoró, pero no alcanzó el nivel del hueso de los ratones alimentados sólo con alimentos duros. diversas maloclusiones que afectan la masticación. Este estudio (Araujo et al, 2014) mostró que la maloclusión de clase I tiene la menor cantidad de ineficiencia de mascar, seguido por la clase II y, finalmente, por la clase III. Así que, cuando la gravedad de la oclusión incrementa, disminuye la eficiencia de mascar. Y un artículo por Huh y otros (2013) apoyan la regulación epigenética del 
fenotipo muscular.

¿Cómo fue que resultamos con tantas personas en los EE.UU. que requieren tratamiento de ortodoncia a causa de maloclusión significativa? Y ahora la cirugía parece ser también una solución aceptable para la maloclusión en los adolescentes. ¿Cómo estamos acelerando klinorynchy en la sociedad norteamericana (y en la sociedad europea en un grado similar? Posiblemente todo empieza muy temprano en la vida, con las prácticas modernas del destete, y con las comidas blandas para los bebés.

Por razones obvias, no podemos hacer estudios en los huesos y los músculos de los niños, asi, que una vez más nos fijamos en las características histológicas en otros animales. Un estudio (Suzuki et al, 2007) muestra cómo la estructura de los músculos temporales cambian tan pronto como los ratones son destetados. Y un estudio de Okubo et al, 2006 muestra cómo la posición y composición del digástrico anterior cambia cuando la masticación y la deglución van de líquido a sólido durante la fase del destete.

Los músculos de la lengua también cambian durante el período del destete, adquiriendo más cadena pesada de miosina (que es un proceso epigenético), para lidiar mejor con la comida sólida (Maejima et al, 2005). Y las glándulas salivales cambian también, cuando el animal (y muy probable los niños también) cambian de la leche a una alimentación regular (Elewa et al, 2014). Entonces, es posible que nosotros estamos quizá privando a los niños pequeños de la unidad de epigenética para crear huesos mejores y más fuertes, dándoles la comida del bebé, y luego alimentos suaves y comida rápida.

Pero los efectos epigenéticos no sólo están presentes en la infancia. Un estudio por Kimura et al, 2006 sugieres que cuando las funciones orales como la masticación y la deglución se omiten por un tubo nasogástrico o un tubo $G$, todo el sistema digestivo empieza a deteriorarse $y$, a menudo sin posibilidad de recuperación a la condición anterior, ya que el cerebro no es capaz de procesar fácilmente algo que nunca antes había sucedido a los seres humanos, tales como la alimentación vía parenteral. Parece que se la boca dice "no hay comida" el cerebro dice: ¿no hay comida? Entonces no hay necesidad de un sistema digestivo."

Un otro estudio (Lunding et al., 2010), realizado en un grupo de personas que se les dió sopa clara o sopa clara y goma de mascar. El grupo que recibió la goma de mascar presentó una mejor movilidad del estómago y menos dispepsia, comparado con el grupo que sólo recibió la sopa. Así que tan solo masticar podría sugerirse que se añade como una solución para algunos problemas digestivos. Y esto sin tomar en cuenta los estudios sobre las relaciones entre, la masticación y el estrés (Gomez et al, 2010; Hori et al, 2005; Koizumi et al, 2011), la pobre masticación y la obesidad (Shiozawa, 2015; Isabel et al, 2015; Zhu \& Hollis, 2015) la masticación y la atención y el rendimiento (Hodoba, 1999; Kutoba et al, 2003; Hasegawa et al, 2007; Kawanishi et al, 2010; Hirano et al, 2012; Ono et al, 2010).

Se puede usar masticación terapéutica en pacientes con disturbios de la ATM o trastornos del sueño, y con dolor de la cara, sabiendo que el dolor es muscular y, a menudo se acompaña de maloclusión y cambios en el estado de ánimo e incluso cambios en el patrón de sueño, que a su vez agrava el dolor. La terapia puede incluir la conciencia de todos las parafunciones que se desarrollan como mecanismo compensatorio, y masticar con una oblea de silicona o un tubo de látex (sham chewing) o goma de mascar (chewing gum). La falta de mascar o comer con alimentos blandos por muchos meses después de cirugía maxillofacial necesita una terapia rápida y cuidadosa. La falta de mascar puede comprometer la oclusión de los dientes y luego la estabilidad de la mandíbula y la estabilidad de los tratamientos de ortodoncia o cirugía. A veces, la falta de mascar correctamente, 
depende de la respiración nasal. El control de alergias y resolución de los trastornos anatómicos de la nariz es la mejor terapia.

Entonces, los principios de la epigenética explican el crecimiento y desarrollo de los huesos y los músculos. La matriz funcional es el concepto que explica cómo el efecto epigenético actualmente envuelve a los dos grupos de tejidos blandos y duros. El mejor efecto epigenético está dado por la masticación, ya que masticar impacta directamente al hueso. Nuevos estudios están apoyando este concepto y probablemente muchos más serán publicados pronto.

\section{REFERENCIAS BIBLIOGRÁFICAS}

Varrela J. Dimensional variation of craniofacial structures in relation to changing masticatoryfunctional demands. Eur J Orthod. 1992 Feb;14(1):31-6.

Lieberman D. 2011. The evolution of the human head. Belknap Harvard, Cambridge, Massachusetts.

Schumacher KU, Koppe T, Fanghänel J, Schumacher GH, Nagai H. Morphometric studies on the facial skeleton of humans and pongids based on CT-scans. Kaibogaku Zasshi. 1994 Oct;69(5):63644.

Francis RC. 2011. Epigenetics: How environment shapes our genes. WW Norton, London.

Hallgrimsson B \& Hall B (edited by) Epigenetics: Linking Genotype and Phenotype in Development and Evolution edited by Benedikt Hallgrimsson and Brian K. Hall (2011). University of California Press, Los Angeles, California.

Corruccini RS. 1984. An epidemiological transition in dental occlusion in world population. Am J Orthodontics Dentofacial Orthopaedics, 86: 419-26.

Corruccini RS, Townsend GC, Richard LC, Brown T. 1990. Genetics and environmental determinants of dental occlusal variation in twins of different nationality. Human Biology, 122:353-67.

Moss ML. 1997. The functional matrix hypothesis revisited. 1. The role of mechanotransduction. Am J Orthod Dentofac Orthop;112:8-11.

Moss ML.1997. The functional matrix hypothesis revisited. 2. The role of an osseous connected cellular network. Am J Orthod Dentofac Orthop 1997;112:221-6.

Moss ML. 1997. The functional matrix hypothesis revisited. 3. The genomic thesis. Am J Orthod Dentofac Orthop 1997;112:338-42.)

Moss ML. 1997. The functional matrix hypothesis revisited. 4. The epigenetic antithesis and the resolving synthesis. Am J Orthod Dentofac Orthop 1997;112:410-7.

Katsaros C, Zissis A, Bresin A \& Killaridis S. 2006. Functional influence on sutural bone apposition in the growing rat. Am J Orthod Dentofacial Orthop. Mar 129(3):352-7.

Mavropoulos A, Odman A, Ammann P, Killaridis S. 2010. Rehabilitation of masticatory function improves the alveolar bone architecture of the mandible in adult rats. Bone; Sep 47(3):687-92. doi: 10.1016/j.bone.2010.06.025. 
Araujo SC, Vieira MM, Gasparotto CA, and Bommarito S. Análise da força de mordida nos diferentes tipos de maloclusões dentárias, segundo Angle. Rev. CEFAC [online]. 2014, vol.16, n.5, pp. 1567-1578. ISSN 1982-0216. http://dx.doi.org/10.1590/1982-021620145113.

Huh A, Horton MJ, Cuenco KT, Raoul G, Rowlerson AM, Ferri J, Sciote JJ. Epigenetic influence of KAT6B and HDAC4 in the development of skeletal malocclusion. Am J Orthod Dentofacial Orthop. 2013 Oct;144(4):568-76. doi: 10.1016/j.ajodo.2013.06.016.

Suzuki K, Abe S, Kim HJ, Usami A, Iwanuma O, Okubo H, Ide Y. 2007. Changes in the muscle fibre properties of the mouse temporal muscle after weaning. Anat Histol Embryol. Apr;36(2):103-6.

Okubo K, Abe S, Usami A, Agematsu H, Nakamura H, Hashimoto M, Ide Y. 2006. Changes in muscle-fiber properties of the murine digastric muscle before and after weaning. Zoolog Sci. Dec;23(12):1079-84.

Maejima M1, Abe S, Sakiyama K, Agematsu H, Hashimoto M, Tamatsu Y, Ide Y. 2005. Changes in the properties of mouse tongue muscle fibres before and after weaning. Arch Oral Biol. Dec;50(12):988-93.

Elewa YH1, Ichii O, Otsuka S, Hashimoto Y, Kon Y. 2014. Structural changes of goat parotid salivary gland: pre- and post-weaning periods. Anat Histol Embryol. Aug;43(4):265-72. doi: 10.1111/ ahe.12071. Epub 2013 Jul 5.

Kimura $Y$ et al. 2006. Evaluation of the effects of mastication and swallowing on gastric motility using electrogastrography. J Med Invest; Aug 53(3-4-):229-37.

Lunding JA, Nordström LM, Haukelid AO, Gilja OH, Berstad A, Hausken T. Vagal activation by sham feeding improves gastric motility in functional dyspepsia. Neurogastroenterol Motil. 2008 Jun;20(6):618-24. doi: 10.1111/j.1365-2982.2007.01076.x. Epub 2008 Jan 31.

Gomez, FM et al. 2010. Relationship between non-functional masticatory activity and central dopamine in stressed rats. J Oral Rehabil; 37:827-833.

Hori $\mathrm{N}$ et al. 2005. Suppression of Stress-induced nNOS Expression in the Rat Hypothalamus by Biting. J Dent Res; 84(7):624-628.

Koizumi et al. 2011. Chewing reduces sympathetic nervous response to stress and prevents poststress arrhythmias in rats. Am J Phisiol Heart Circ Physiol; 301(4):H1551-8. doi: 10.1152/ ajpheart.01224.2010.

Shiozawa K, Ohnuki Y, Mototani Y, Umeki D, Ito A, Saeki Y, Hanada N, Okumura S. Effects of food diameter on bite size per mouthful and chewing behavior. J Physiol Sci. 2015 Oct 22. [Epub ahead of print] masticatory and swallowing behaviors and body weight. Physiol Behav. 2015 Nov 1;151:314-9. doi: 10.1016/j.physbeh.2015.08.006. Epub 2015 Aug 4.

Zhu Y, Hollis JH. Relationship between chewing behavior and body weight status in fully dentate healthy adults. Int J Food Sci Nutr. 2015 Mar;66(2):135-9. doi: 10.3109/09637486.2014.979317. Epub 2015 Jan 13. 
Hodoba, D. (1999). Chewing can relieve sleepiness in a night of sleep deprivation. Sleep Research Online 2: 101-105. http://www.sro.org./1999/Hodoba/101/

Kutoba K et al. 2003. Nuclear medical PET-study in the causal relationship between mastication and brain function in human evolutionary and developmental processes. Ann Anat. Dec;185(6):565-9.

Hasegawa Y, Ono T, Hori K \& Nobuki T. 2007. Influence of Human Jaw Movement on Cerebral Blood Flow J Dent Res 86(1):64-68.

Kawanishi K, Koshino H, Toyoshita Y, Tanaka M, Hirai T. 2010. Effect of mastication on functional recoveries after permanent middle cerebral artery occlusion in rats. J Stroke Cerebrovasc Dis. 2010 Sep-Oct;19(5):398-403. doi: 10.1016/j.jstrokecerebrovasdis.2009.07.011. Epub 2010 May 15.

Hirano Y, Obata T, Takahashi H, Tachibana A, Kuroiwa D, Takahashi T, Ikehira H, Onozuka M. 2013. Effects of chewing on cognitive processing speed. Brain Cogn. Apr;81(3):376-81. doi: 10.1016/j. bandc.2012.12.002. Epub 2013 Jan 29.

Ono Y, Yamamoto T, Kubo KY, Onozuka M. 2010. Occlusion and brain function: mastication as a prevention of cognitive dysfunction. J Oral Rehabil. Aug;37(8):624-40. doi: 10.1111/j.13652842.2010.02079.x. Epub 2010 Mar 2. 